\title{
Helicobacter pylori induces epithelial-mesenchymal transition in gastric carcinogenesis via the AKT/GSK3ß signaling pathway
}

\author{
YAOBIN OUYANG $^{1 *}$, GONGMEIZI LIU ${ }^{1,2^{*}}$, WENTING XU ${ }^{1,3}$, ZHEN YANG $^{1 \#}$, NIANSHUANG LI ${ }^{1}$, \\ CHUAN XIE $^{1}, \mathrm{CHUN} \mathrm{ZHOU}^{4}$, JIANG CHEN ${ }^{1}$, YIN ZHU ${ }^{1}$, JUNBO HONG ${ }^{1}$ and NONGHUA LU ${ }^{1}$ \\ ${ }^{1}$ Department of Gastroenterology, The First Affiliated Hospital of Nanchang University, Nanchang, Jiangxi 330006; \\ ${ }^{2}$ Department of Endocrinology, The First Affiliated Hospital of Tsinghua University, Beijing 100700; \\ ${ }^{3}$ Department of Gastroenterology, The First Affiliated Hospital, College of Medicine, Zhejiang University, Hangzhou, \\ Zhejiang 310003; ${ }^{4}$ Department of Urology, The Haidian Hospital of Beijing, Beijing 100700, P.R. China
}

Received April 28, 2020; Accepted October 19, 2020

DOI: $10.3892 / \mathrm{ol} .2021 .12426$

\begin{abstract}
Helicobacter pylori (H. pylori) is a main risk factor for gastric cancer (GC). Epithelial-mesenchymal transition (EMT) is involved in the development and progression of H. pylori-associated GC. However, the exact molecular mechanism of this process remains unclear. The AKT/GSK3 $\beta$ signaling pathway has been demonstrated to promote EMT in several types of cancer. The present study investigated whether H. pylori infection induced EMT, and promoted the development and metastasis of cancer in the normal gastric mucosa, and whether this process was dependent on AKT activation. The expression levels of the EMT-associated proteins, including E-cadherin and $\mathrm{N}$-cadherin, were determined in 165 gastric mucosal samples of different disease stages by immunohistochemical analysis. The expression levels of E-cadherin, N-cadherin, AKT, phosphorylated (p-) AKT (Ser473), GSK3 $\beta$ and p-GSK3 $\beta$ (Ser9) were further determined in $H$. pylori-infected Mongolian gerbil gastric tissues and cells co-cultured with $H$. pylori by immunohistochemical analysis and western blotting. The results indicated that the expression levels of the epithelial marker E-cadherin were decreased, whereas the expression levels of the mesenchymal marker $\mathrm{N}$-cadherin were increased during gastric carcinogenesis. Their expression levels were associated with $H$. pylori infection. Furthermore, H. pylori infection resulted
\end{abstract}

Correspondence to: Dr Junbo Hong or Dr Nonghua Lu, Department of Gastroenterology, The First Affiliated Hospital of Nanchang University, 17 Yongwaizheng Street, Nanchang, Jiangxi 330006, P.R. China

E-mail: doctorhjb@126.com

E-mail: lunonghua@ncu.edu.cn

"Deceased

*Contributed equally

Key words: gastric carcinogenesis, Helicobacter pylori, epithelial-mesenchymal transition, AKT/GSK3 $\beta$ pathway in downregulation of E-cadherin expression and upregulation of N-cadherin expression in Mongolian gerbils and GES-1 cells. In addition, an investigation of the associated mechanism of action revealed that p-AKT (Ser473) and p-GSK3 $\beta$ (Ser9) were activated in GES-1 cells following co-culture with H.pylori.Furthermore, following pretreatment of the cells with the AKT inhibitor VIII, the expression levels of E-cadherin, $\mathrm{N}$-cadherin, $\mathrm{p}$-AKT and p-GSK3 $\beta$ did not show significant differences between GES-1 cells that were co-cultured with or without $H$. pylori. The levels of p-AKT and p-GSK3 $\beta$ were increased in $H$. pylori-infected Mongolian gerbils. In conclusion, the present study demonstrated that $H$. pylori infection activated AKT and resulted in the phosphorylation and inactivation of GSK3 $\beta$, which in turn promoted early stage EMT. These effects were AKT-dependent. This mechanism may serve as a prerequisite for GC development.

\section{Introduction}

Although its incidence is declining, gastric cancer (GC) is still a leading cause of cancer-associated mortality and a major socio-economic burden worldwide (1). Helicobacter pylori (H. pylori) infection is a major risk factor of GC (2). Environmental factors are subordinate to the effect of $H$. pylori infection, which has been listed as a class I carcinogen for GC by the World Health Organization since 1994 (2). H. pylori infection causes gastric mucosal inflammation, as well as genetic and epigenetic changes, which lead to genetic instability in host gastric epithelial cells (2). However, the exact molecular mechanism of $H$. pylori-associated GC is not yet clear (3). At present, several studies have demonstrated that $H$. pylori induces the epithelial-mesenchymal transition (EMT) process in gastric epithelial cells, which promotes invasion and metastasis of GC via the TGF- $\beta, N F-\kappa B$ and Hippo signaling pathways (4-6).

EMT is a process in which epithelial cells lose their polarity and transform their morphology by developing fiber-like structures (7). Simultaneously, the contacts with the surrounding cells and cell adhesion are reduced and an increased migratory and invasive activity is noted. Both of these processes promote tumor metastasis (7). The molecular 
changes of EMT are mainly characterized by the downregulation of the expression of epithelial cell markers and the upregulation of the expression of mesenchymal phenotypic markers (8). In particular, the absence of E-cadherin is an important EMT signal and a prerequisite for tumor cell invasion (9). The functional molecules of mesenchymal cells are mainly skeletal proteins derived from mesenchyme or embryos (e.g., N-cadherin) that are normally not expressed or expressed at low levels in epithelial cells, while the expression of these molecules is increased during the EMT process (10).

Multiple signaling pathways are involved in the development of EMT (11). For example, hepatocyte growth factor/scatter factor and early growth response gene 1 synergistically activate the MAPK signaling pathway, which enhances the activity of snail and inhibits the expression of the epithelial marker E-cadherin, promoting EMT (12). The AKT/GSK3 $\beta$ signaling pathway has attracted considerable attention due to its involvement in EMT (13). AKT, which is also known as protein kinase $\mathrm{B}$, is a serine/threonine protein kinase associated with a number of signaling pathways that promote cell proliferation, survival and inhibition of apoptosis (14). GSK3 $\beta$ is a serine/threonine protein kinase that acts downstream of AKT (15). Unlike other kinases, GSK3 $\beta$ is normally dephosphorylated in its active state. The phosphorylation of GSK3 $\beta$ (Ser9) by phosphorylated (p-)AKT leads to inhibition of its activity (15). Activation of AKT results in GSK3 $\beta$ phosphorylation, which reduces $\beta$-catenin degradation, promotes Snail expression and eventually induces EMT in combination with $\mathrm{T}$ cell factor/lymphocyte enhancer factor $(13,16)$.

Previous studies demonstrated that $H$. pylori activated the AKT signaling pathway in human gastric epithelial cells (GES-1), thereby causing disorders in cell proliferation and apoptosis $(17,18)$. However, to the best of our knowledge, the role of the AKT/GSK3 $\beta$ signaling pathway in H. pylori-associated EMT is not yet clear. The aim of the present study was to investigate whether $H$. pylori infection induces EMT and promotes the development and metastasis of cancer in normal gastric mucosa. In addition, the present study investigated whether this process was dependent on AKT activation. The pathogenic mechanism of $H$. pylori was thoroughly examined and the data provided a novel theoretical basis for the development of prevention strategies for $H$. pylori infection-associated diseases.

\section{Materials and methods}

Patients and sample collection. Between January 2007 and September 2012, 165 formalin-fixed (10\% formalin at room temperature for $24 \mathrm{~h}$ ) and paraffin-embedded gastric tissue samples from 165 patients who underwent gastroduodenoscopy were obtained from the Digestive Centre of The First Affiliated Hospital of Nanchang University (Nanchang, China). Patients aged between 18-70 years and without previous $H$. pylori treatment history were enrolled in the present study, and patients who underwent gastric surgery were excluded. The clinical characteristics of patients were stratified according to pathological diagnosis and are shown in Table I. The patients had a median age of 55 years (range, 36-69 years) and 86 patients $(52 \%)$ were male. Among these patients, 19 out of the 39 who were diagnosed with chronic non-atrophic gastritis (CNAG),
19 out of the 40 who had intestinal metaplasia (IM), 19 out of the 39 who had dysplasia (Dys) and 22 out of the 47 who had GC were $H$. pylori-positive. The age and gender distribution of these patients were compared and the data revealed no significant differences ( $\mathrm{P}>0.05$; Table I). Gastric tissue samples were collected from the gastric antrum and from the individual lesions of each patient. Pathological diagnosis and classification were based on the criteria of the World Health Organization and the updated Sydney system $(19,20)$. A non-atrophy gastritis diagnosis was made when all biopsies indicated a non-atrophy gastritis result $(19,20)$. The rapid urease test and Giemsa staining were used for the detection of H. pylori infection, as previously described (21). The present study was approved by the Human Ethics Committee of the First Affiliated Hospital of Nanchang University (Nanchang, China). All patients signed an informed consent form for their participation in the study.

H.pylori strain and culture conditions. The wild-type H.pylori $\mathrm{CagA}^{+} \mathrm{VaCA}^{+}$strain (ATCC43504; American Type Culture Collection) was used in the present study. Briefly, H. pylori was cultured on Campylobacter agar (Oxoid; Thermo Fisher Scientific, Inc.) plates with $5 \%$ sheep blood (Gibco; Thermo Fisher Scientific, Inc.) and incubated at $37^{\circ} \mathrm{C}$ with $5 \% \mathrm{CO}_{2}$ for subsequent in vitro experiments. Following $24 \mathrm{~h}$ of incubation, H. pylori strains were grown in Brucella broth (Gibco; Thermo Fisher Scientific, Inc.) with $10 \%$ sheep serum (Gibco; Thermo Fisher Scientific, Inc.) for $16 \mathrm{~h}$ under microaerophilic conditions $\left(5 \% \mathrm{O}_{2}, 10 \% \mathrm{CO}_{2}\right.$ and $\left.85 \% \mathrm{~N}_{2}\right)$. H. pylori co-culture experiments with GES-1 cells were conducted at an MOI of $50: 1$.

Animals and infections. Male Mongolian gerbils (specific pathogen-free; age, 5-8 weeks; weight, 30-50 g; obtained from Zhejiang Academy of Medical Sciences (Zhejiang, China) http://www.zjams.com.cn/) were utilized in the present study between August 2018 and June 2019. A total of 79 gerbils were maintained in an isolated clean room with regulated temperature $\left(20-22^{\circ} \mathrm{C}\right)$, humidity $(\sim 55 \%)$ and a 12/12-h light/dark cycle, with ad libitum access to rodent diet and water as previously described, animal health and behavior were monitored twice a day, and no gerbils were found dead during experiments (22). Following fasting for $12 \mathrm{~h}$, the animals were orogastrically challenged with $0.2 \mathrm{ml}$ sterile Brucella broth, which was used as a control group $(n=25)$ or H. pylori strains $(n=54)$ at 10 time points (every 3 days). The Mongolian gerbils were humanely euthanized via $\mathrm{CO}_{2}$ inhalation (30\% of the chamber volume per min) at 9 months after the challenge and gastric tissues were harvested. Euthanasia was confirmed by observation of each gerbil for lack of respiration and faded eye color. The First Affiliated Hospital of Nanchang University Ethics Committee (Nanchang, China) approved all experiments and procedures.

Cell line and reagents. The immortalized human gastric cell line GES-1 (Beijing Institute for Cancer Research, Beijing, China) was grown in DMEM (Gibco; Thermo Fisher Scientific, Inc.) with $10 \%$ fetal bovine serum (Gibco; Thermo Fisher Scientific, Inc.) and $1 \%$ penicillin/streptomycin at $37^{\circ} \mathrm{C}$ with $5 \% \mathrm{CO}_{2}$. The AKT inhibitor VIII was purchased from Merch KGaA. 
Table I. Clinical characteristics of patients with different stages of gastric carcinogenesis.

\begin{tabular}{|c|c|c|c|c|c|}
\hline Characteristic & CNAG & IM & Dys & GC & P-value \\
\hline No. & 39 & 40 & 39 & 47 & \\
\hline Age, years $($ mean $\pm S D)$ & $50.80 \pm 14.08$ & $54.32 \pm 12.18$ & $56.68 \pm 10.81$ & $55.06 \pm 14.01$ & $>0.05$ \\
\hline Male, $\mathrm{n}$ & 20 & 21 & 20 & 25 & $>0.05$ \\
\hline Female, $\mathrm{n}$ & 19 & 19 & 19 & 22 & $>0.05$ \\
\hline H. pylori positive, $\mathrm{n}$ & 19 & 19 & 19 & 22 & $>0.05$ \\
\hline H. pylori negative, $\mathrm{n}$ & 20 & 21 & 20 & 25 & $>0.05$ \\
\hline
\end{tabular}

CNAG, chronic non-atrophic gastritis; IM, intestinal metaplasia; Dys, dysplasia; GC, gastric cancer; H. pylori, Helicobacter pylori. One-way ANOVA test (age) or the $\chi^{2}$ test (sex, H. pylori infection status) were used to determine the differences among groups.

Western blotting. GES-1 cells were co-cultured with $H$. pylori strain 43504, and total protein was extracted in RIPA lysis buffer (Beyotime Institute of Biotechnology). Protein concentration was determined using a BCA assay kit (Thermo Fisher Scientific, Inc.). Protein (20 $\mu \mathrm{g} /$ lane) from each sample was separated by $10 \%$ SDS-PAGE and transferred to PVDF membranes, as previously described (21). Following blocking with $5 \%$ non-fat milk at room temperature for $1 \mathrm{~h}$, the blots were incubated with the primary antibodies overnight at $4{ }^{\circ} \mathrm{C}$ and with HRP-conjugated secondary antibodies (dilution, 1:5,000; cat. no. ZB-2301; Beijing Zhongshan Golden Bridge Biotechnology Co., Ltd.) at $4^{\circ} \mathrm{C}$ for $4 \mathrm{~h}$. The protein bands were visualized using the Super-Signal West Femto Maximum Sensitivity Substrate Kit (Thermo Fisher Scientific, Inc.) and exposed by the Bio-Rad Image Lab system (Bio-Rad Laboratories, Inc.). The band intensities of the target proteins were normalized to those of $\beta$-actin using Image Lab software (version 5.2.1; Bio-Rad Laboratories, Inc.). The primary antibodies and their sources were as follows: E-cadherin (anti-E-cadherin antibody; dilution, 1:1,000; cat. no. ab1416; Abcam), N-cadherin (anti-N-cadherin antibody; dilution, 1:1,000; cat. no. ab18203; Abcam), AKT (anti-AKT antibody; dilution, 1:1,000; cat. no. 4691; Cell Signaling Technology, Inc.), p-AKT (Ser473) (anti-p-AKT antibody; dilution, 1:1,000; cat. no. 9271; Cell Signaling Technology, Inc.), GSK3 $\beta$ (anti-GSK3 $\beta$ antibody; dilution, 1:1,000; cat. no. 9315S; Cell Signaling Technology, Inc.), p-GSK3 $\beta$ (Ser9) (anti-p-GSK3 $\beta$ antibody; dilution, 1:1,000; cat. no. 9322; Cell Signaling Technology, Inc.) and $\beta$-actin ( $\beta$-actin antibody; dilution, 1:1,000; cat. no. sc-1615-R; Santa Cruz Biotechnology, Inc.).

Immunohistochemical analysis. Biopsy specimens and animal gastric tissues were processed for immunohistochemical analysis as previously described (21). Briefly, specimens were sectioned at $4-\mu \mathrm{m}$ thickness and baked at $70^{\circ} \mathrm{C}$ for $1.5 \mathrm{~h}$. Subsequently, sections were de-paraffinized in xylene and rehydrated with gradient ethanol (100, 95 and 85\% ethanol). The sections were repaired with citrate buffer solution (TransGen Biotech Co., Ltd.) at $100^{\circ} \mathrm{C}$ for $15 \mathrm{~min}$, and then washed in PBS solution (TransGen Biotech Co., Ltd.) three times. Following blocking with $3 \% \mathrm{H}_{2} \mathrm{O}_{2}$ solution at room temperature for $8 \mathrm{~min}$ and incubation with $5 \%$ goat serum (Beyotime Institute of Biotechnology) at room temperature for $30 \mathrm{~min}$, sections were incubated with the primary antibodies at $4^{\circ} \mathrm{C}$ overnight and with secondary antibodies (dilution, 1:100; $100 \mu 1$ per section; cat. no. PV-6000; Beijing Zhongshan Golden Bridge Biotechnology Co., Ltd.) at $37^{\circ} \mathrm{C}$ for 30 mins. Sections were stained with DAB solution (Beijing Zhongshan Golden Bridge Biotechnology Co., Ltd.) and evaluated under a fluorescence microscope (Olympus bx53 biomicroscope; Olympus Corporation). The samples were stained with anti-E-cadherin antibody (biopsy and gastric tissues; dilution, 1:300; cat. no. ab1416; Abcam), anti-N-cadherin antibody (biopsy and gastric tissues; dilution, 1:800; cat. no. ab18203; Abcam), anti-AKT antibody (gastric tissues; dilution, 1:500; cat. no. ab8805; Abcam), anti-p-AKT antibody (Ser473) (gastric tissues; dilution, 1:500; cat. no. ab66138; Abcam), anti-GSK3 $\beta$ antibody (gastric tissues; dilution, 1:400; cat. no. ab98081; Abcam) and anti-p-GSK3 $\beta$ antibody (Ser9) (gastric tissues; dilution, 1:200; cat. no. ab75814; Abcam). Two pathologists (Department of Pathology, The First Affiliated Hospital of Nanchang University, Nanchang, China) who were blinded to the clinicopathological data scored (0-12 points) and assessed (grade 1-4) the stained sections by evaluating and calculating the intensity (0-3 points) and frequency (0-4 points) of staining. The relative expression levels of the proteins were reported as grades as follows: Grade 1 ( $0-2$ points), grade 2 (3-5 points), grade 3 (6-8 points) and grade 4 (9-12 points) (18). Grading discrepancies were re-reviewed and discussed to obtain a final score.

Statistical analysis. The results are presented as the mean \pm standard deviation of three independent experiments or the percentage compared with the control samples. The $\chi^{2}$ and Mann-Whitney tests were used to analyze the statistical difference between two groups. One-way ANOVA followed by Tukey's post hoc test or the Kruskal-Wallis test followed by Bonferroni post hoc test were used to determine the differences among multiple groups based on the normality of the data (SPSS software; version 18.0; SPSS, Inc.). P $\leq 0.05$ was considered to indicate a statistically significant difference.

\section{Results}

Expression levels of EMT-associated proteins in gastric carcinogenesis. To evaluate the expression levels of EMTassociated proteins in patients with gastric carcinogenesis, an immunohistochemical analysis of human gastric tissues of different gastric lesions was performed. The expression 

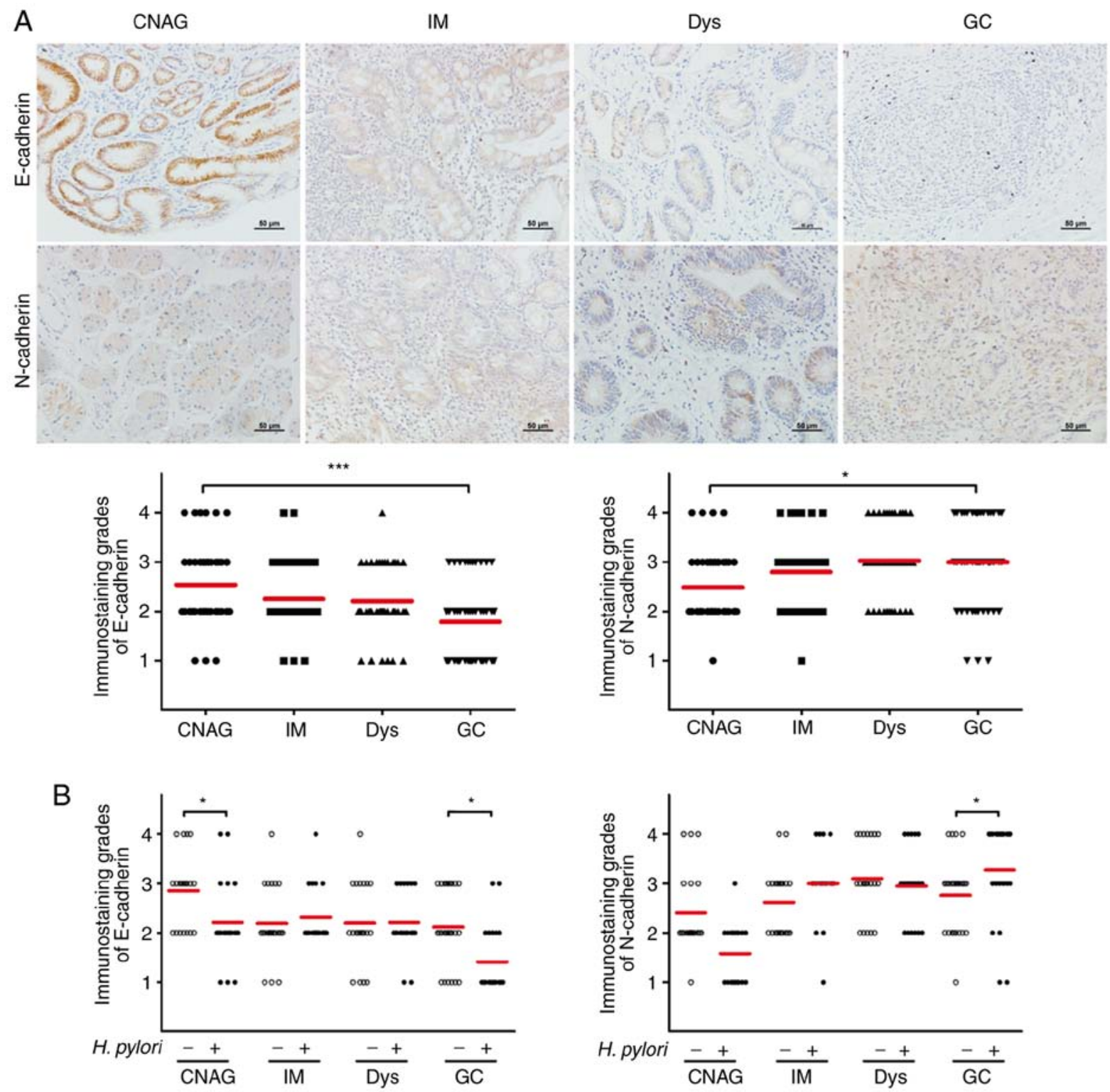

Figure 1. Detection of the expression levels of EMT-associated proteins in gastric carcinogenesis. (A) Expression levels of the EMT-associated markers E-cadherin and $\mathrm{N}$-cadherin in 165 gastric tissue samples at different disease stages (CNAG, IM, Dys and GC) were detected by immunohistochemical analysis. The expression grades were assessed by the staining intensity and frequency. Scale bar, $50 \mu \mathrm{m}$. (B) Expression levels of E-cadherin and N-cadherin were assessed in 165 gastric tissue samples at different disease stages according to $H$. pylori infection status. H. pylori infection was assessed by rapid urease tests and Giemsa staining. ${ }^{*} \mathrm{P}<0.05,{ }^{* * *} \mathrm{P}<0.001$. EMT, epithelial-mesenchymal transition; CNAG, chronic non-atrophic gastritis; IM, intestinal metaplasia; Dys, dysplasia; GC, gastric cancer; H. pylori, Helicobacter pylori.

levels of the epithelial marker E-cadherin were decreased at the IM stage compared with CNAG stage and continued to decrease in the GC stage. E-cadherin expression was significantly reduced in GC tissues compared with in CNAG tissues $(\mathrm{P}<0.001)$. However, the expression levels of the mesenchymal marker $\mathrm{N}$-cadherin were increased at the IM stage compared with CNAG stage and continued to increase in the subsequent stages of gastric carcinogenesis. $\mathrm{N}$-cadherin expression was significantly increased in GC tissues compared with in CNAG tissues $(\mathrm{P}<0.05$; Fig. 1A). These results indicated that EMT may be involved in the initiation of the early stage of gastric carcinogenesis.

H. pylori is a known important risk factor that triggers gastric carcinogenesis (2). The association between $H$. pylori infection and the expression levels of E-cadherin and
$\mathrm{N}$-cadherin was examined in the patients. The immunohistochemical results indicated that the expression levels of E-cadherin were significantly lower following $H$. pylori infection compared with those of non-infected patients $(\mathrm{P}<0.05)$ in $\mathrm{CNAG}$ and $\mathrm{GC}$ stage, whereas the expression levels of $\mathrm{N}$-cadherin were significantly higher following H. pylori infection compared with those of non-infected patients $(\mathrm{P}<0.05$; Fig. 1B) in GC stage. There was no significant difference in E-cadherin and $\mathrm{N}$-cadherin expression in IM and Dys stages. Overall, these results suggested that $H$. pylori infection may induce EMT.

H. pylori infection induces EMT in GES-1 cells and Mongolian gerbils. The initial observation demonstrated an association between $H$. pylori infection and the expression 
A
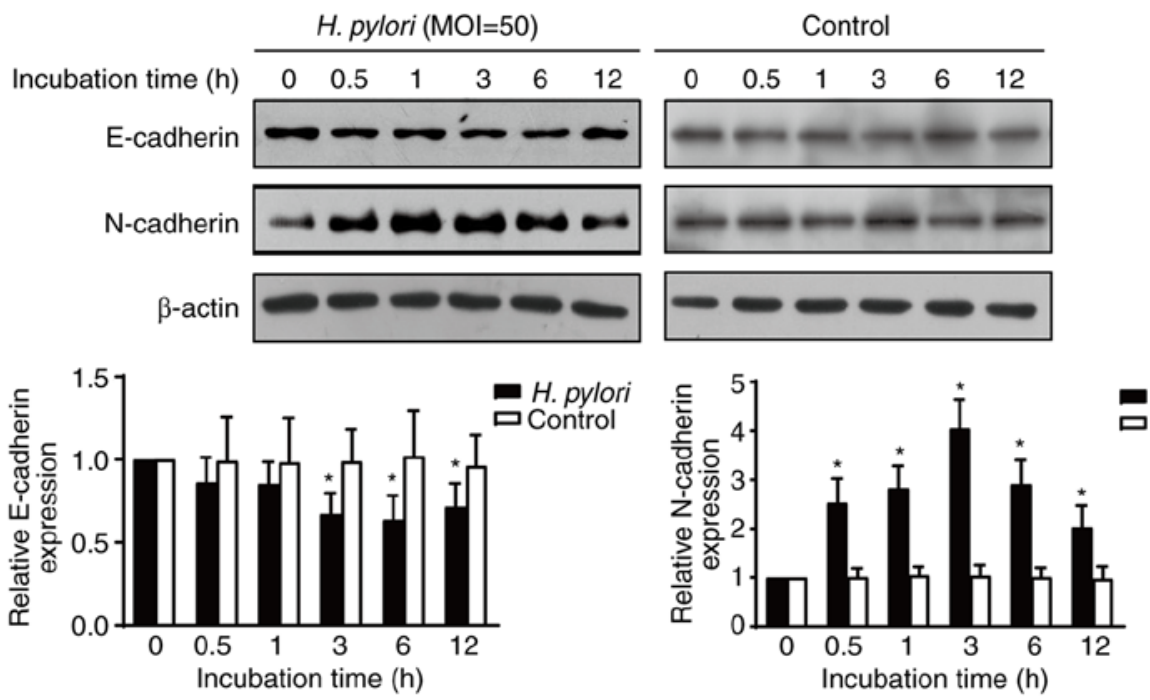

B
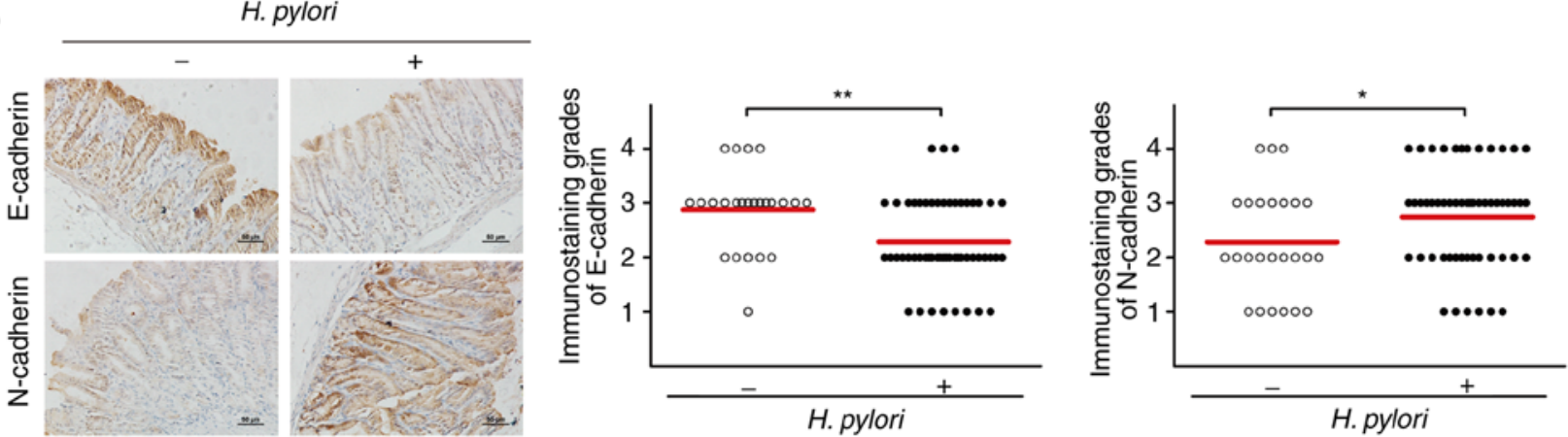

Figure 2. H. pylori infection induces EMT in GES-1 cells and Mongolian gerbils. (A) Expression levels of the EMT-associated markers E-cadherin and $\mathrm{N}$-cadherin were detected by western blot analysis in GES-1 cells following $0,0.5,1,3,6$ and $12 \mathrm{~h}$ of co-culture with or without $H$. pylori. The statistical analysis of three independent experiments is presented. (B) Expression levels of the EMT-associated markers E-cadherin and N-cadherin were detected by immunohistochemical analysis of the gastric mucosa of Mongolian gerbils with or without $H$. pylori infection. The expression grades were assessed by the intensity and frequency of staining. Scale bar, $50 \mu \mathrm{m} .{ }^{*} \mathrm{P}<0.05,{ }^{* *} \mathrm{P}<0.01 \mathrm{vs} .0 \mathrm{~h}$ or as indicated. EMT, epithelial-mesenchymal transition; $H$. pylori, Helicobacter pylori.

levels of EMT-associated markers in human gastric tissues. Subsequently, the effects of $H$. pylori infection on EMT were investigated in gastric epithelial GES-1 cells and in an animal model. GES-1 cells were co-cultured with $H$. pylori and cellular proteins were extracted at different time points. Protein detection was performed by western blotting. The results demonstrated that $H$. pylori infection significantly decreased the expression levels of E-cadherin following $3 \mathrm{~h}$ of co-culture, whereas it increased the expression levels of $\mathrm{N}$-cadherin following $0.5 \mathrm{~h}$ of co-culture $(\mathrm{P}<0.05$; Fig. $2 \mathrm{~A})$. Subsequently, immunohistochemical analysis was performed in order to examine the expression levels of E-cadherin and $\mathrm{N}$-cadherin in gastric tissues of Mongolian gerbils following $H$. pylori infection and in the corresponding tissues of the control group. H. pylori infection significantly increased the expression levels of $\mathrm{N}$-cadherin and decreased the expression levels of E-cadherin $(\mathrm{P}<0.05$; Fig. 2B). In summary, these in vivo and in vitro data demonstrated that $H$. pylori infection may induce EMT.

H. pylori-induced EMT is regulated via the AKT/GSK3 $\beta$ signaling pathway. The molecular mechanisms of $H$. pylori infection were investigated with regard to EMT induction. The AKT/GSK3 $\beta$ signaling pathway has previously been demonstrated to induce EMT (13). To investigate whether the AKT/GSK3 $\beta$ signaling pathway was involved in $H$. pylori-induced EMT, the effects of $H$. pylori infection on the AKT/GSK3 $\beta$ signaling pathway were further investigated in GES-1 cells. Western blot analysis demonstrated that the relative expression ratio of p-AKT (p-AKT/AKT) was significantly increased in GES-1 cells following incubation of the cells with $H$. pylori for $0.5,1,3$ and $6 \mathrm{~h}$, and no significant difference was observed at $12 \mathrm{~h}$ compared with in cells in the group without $H$. pylori incubation $(\mathrm{P}<0.05$; Fig. $3 \mathrm{~A})$. The relative expression ratio of $\mathrm{p}-\mathrm{GSK} 3 \beta$ (p-GSK3 $\beta /$ GSK3 $\beta$ ) was significantly increased in GES-1 cells that were incubated with H.pylori for 3,6 and $12 \mathrm{~h}$ compared with in cells in the group without $H$. pylori incubation ( $\mathrm{P}<0.05$; Fig. 3A).

The role of AKT in H.pylori-induced EMT was further explored. As shown in Figs. 2 and 3, H. pylori-infected GES-1 cells exhibited a prominent increase in the levels of $\mathrm{N}$-cadherin, $\mathrm{p}$-AKT and p-GSK3 $\beta$, whereas a decrease was noted in the expression levels of E-cadherin compared with the corresponding expression levels of the control group $(\mathrm{P}<0.05)$. The results were similar in GES-1 cells that were pre-treated with DMSO. H. pylori infection did not result in a change in the expression levels of E-cadherin, $\mathrm{N}$-cadherin, $\mathrm{p}$-AKT and p-GSK3 $\beta$ in GES- 1 cells that were pre-treated with the AKT 
A

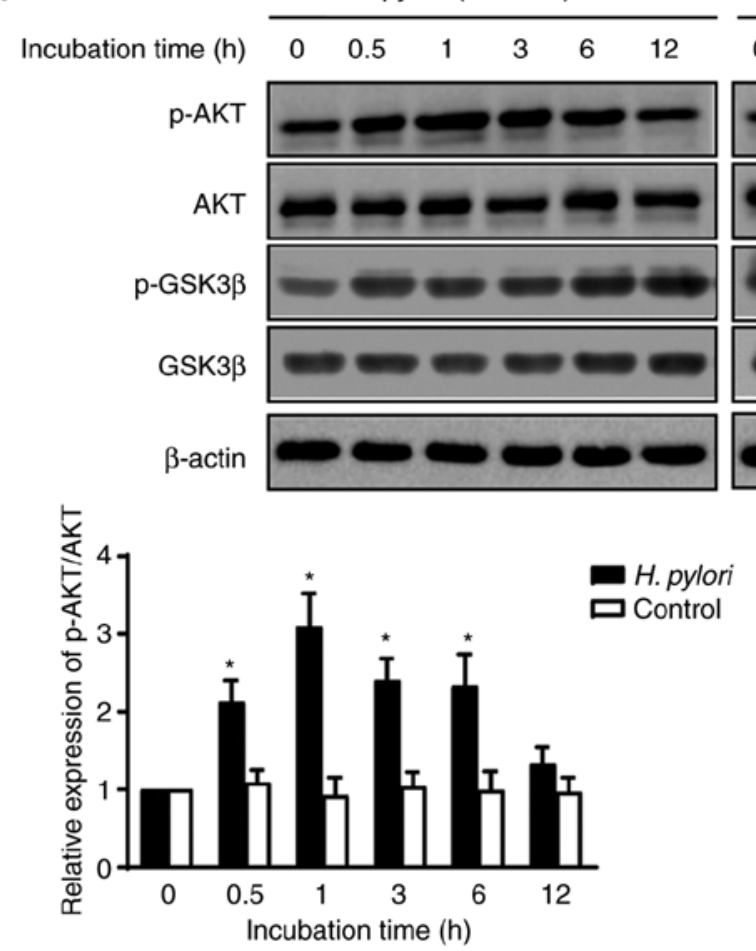

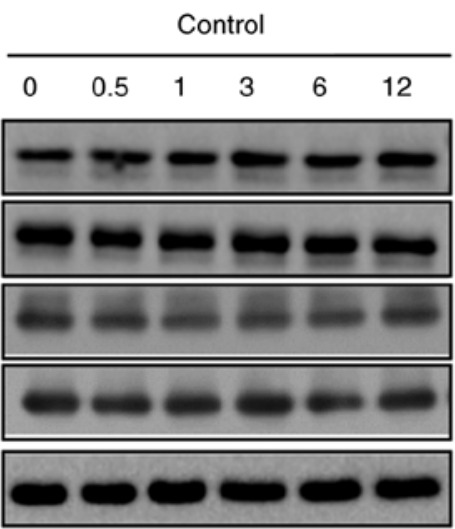

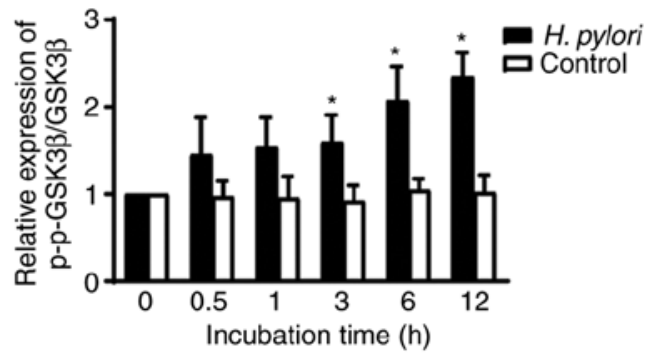

B
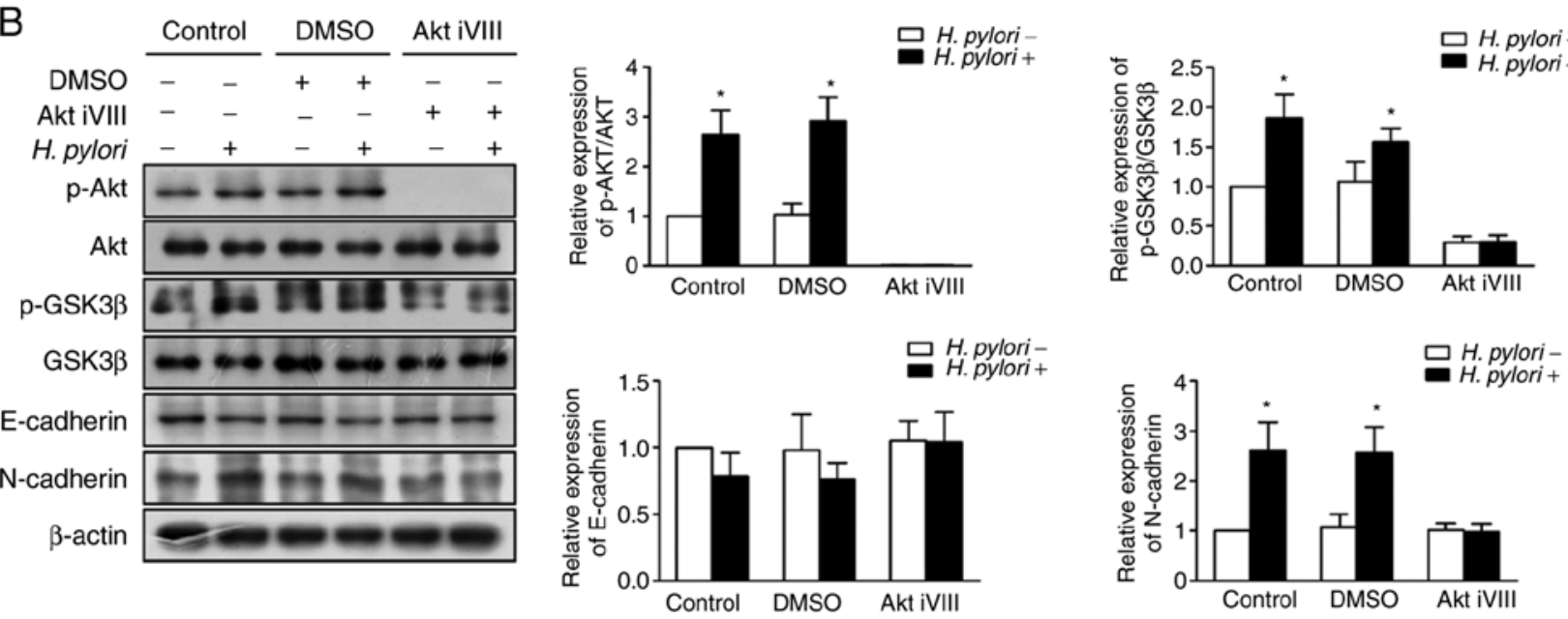

Figure 3. H. pylori-induced epithelial-mesenchymal transition is regulated via the AKT-GSK3 $\beta$ signaling pathway. (A) Expression levels of the AKT-GSK3 $\beta$ signaling pathway-associated proteins AKT, p-AKT (Ser473), GSK3 $\beta$ and p-GSK3 $\beta$ (Ser9) were detected by western blotting in GES-1 cells following 0, $0.5,1,3,6$ and $12 \mathrm{~h}$ co-culture with or without $H$. pylori. The statistical analysis of three independent experiments is shown. (B) GES-1 cells were pretreated with DMSO, the Akt iVIII or control medium. The expression levels of the AKT-GSK3 $\beta$ signaling pathway-associated proteins were detected by western blot analysis in GES-1 cells with or without $H$. pylori infection. The statistical analysis of three independent experiments is shown. ${ }^{*} \mathrm{P}<0.05 \mathrm{vs} .0 \mathrm{~h}$ in $(\mathrm{A})$ and H. pylori (-) group in (B). Akt iVIII, AKT inhibitor VIII; H. pylori, Helicobacter pylori; p-, phosphorylated.

inhibitor VIII (P>0.05; Fig. 3B). Therefore, H. pylori-induced EMT may be blocked by AKT inhibition, suggesting that H.pylori-induced EMT may be regulated by the AKT/GSK3 $\beta$ signaling pathway.

$A K T$ and GSK3 $\beta$ are activated in $H$. pylori-infected Mongolian gerbils. To further validate the role of the AKT/GSK3 $\beta$ signaling pathway in $H$. pylori-induced EMT, immunohistochemical analysis was performed to assess the expression levels of AKT, p-AKT, GSK3 $\beta$ and p-GSK3 $\beta$ in gastric tissues of Mongolian gerbils following $H$. pylori infection and they were compared with those of the control group. Immunohistochemical assays indicated that although the expression levels of AKT and GSK3 $\beta$ did not exhibit significant differences between the two groups $(P>0.05)$, $H$. pylori infection significantly increased the expression levels of p-AKT and p-GSK $3 \beta$ (P<0.05; Fig. 4). Overall, these results further validated the role of the AKT/GSK3 $\beta$ signaling pathway in $H$. pylori-induced EMT.

\section{Discussion}

H. pylori infection is the main risk factor for GC and is responsible for the initiation of gastric carcinogenesis in 


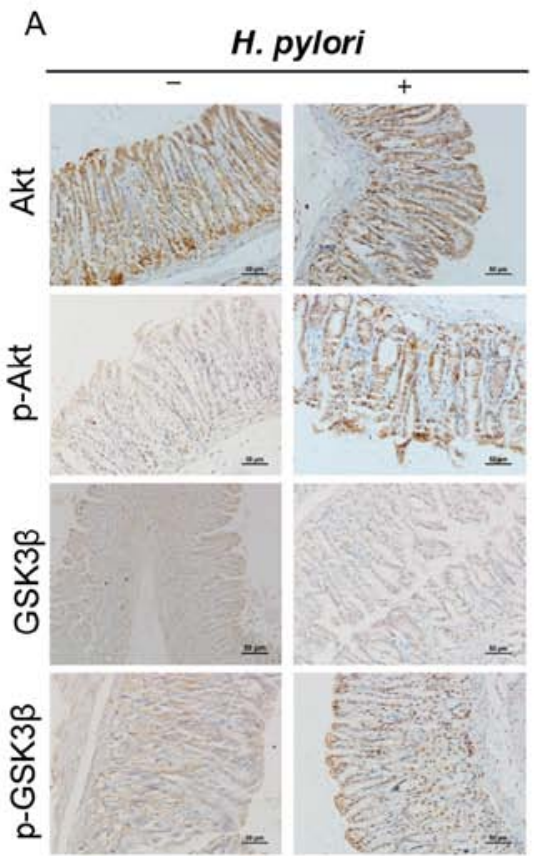

B
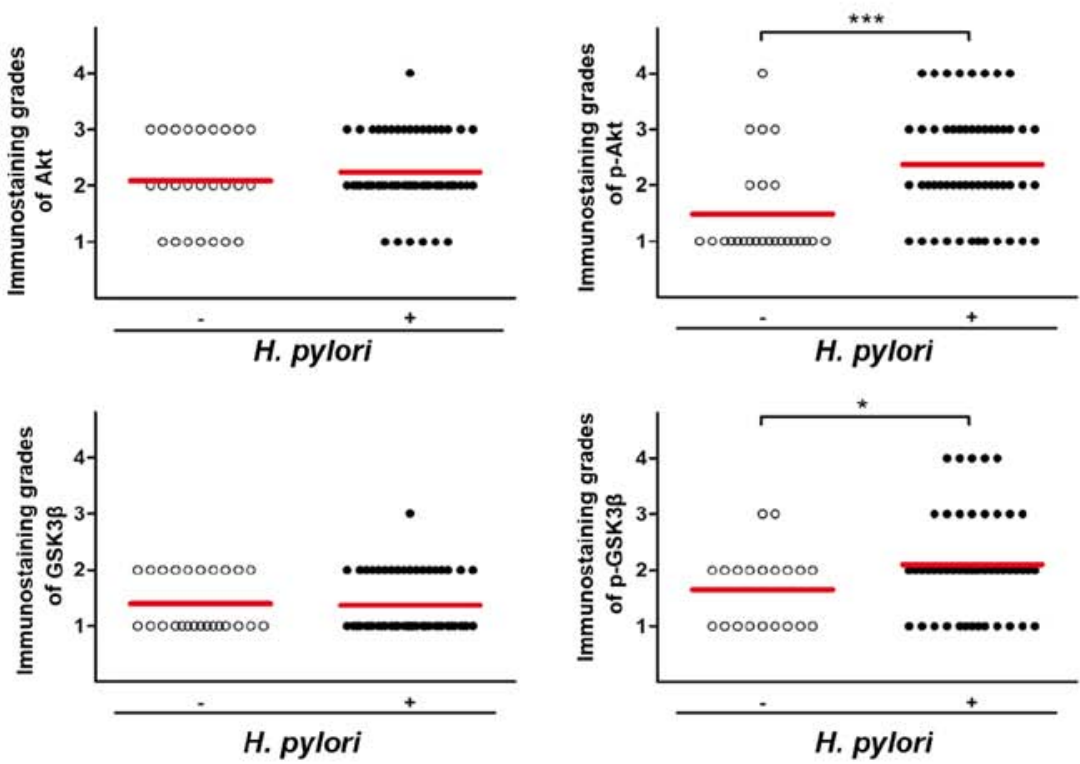

Figure 4. AKT-GSK3 $\beta$ signaling pathway activation in H. pylori-infected Mongolian gerbils. (A) Protein expression levels of the AKT, p-AKT (Ser473), GSK3 $\beta$ and p-GSK3 $\beta$ (Ser9) were detected by immunohistochemical analysis in the gastric mucosa of Mongolian gerbils with or without $H$. pylori infection. Scale bar, $50 \mu \mathrm{m}$. (B) Expression grades were assessed by the staining intensity and frequency. ${ }^{*} \mathrm{P}<0.05,{ }^{* * *} \mathrm{P}<0.001$. H. pylori, Helicobacter pylori; p-, phosphorylated.

different developmental stages, including CNAG, IM, Dys and eventually GC (23). It has been reported that various oncogenic signaling pathways are involved in the pathogenic effects of $H$.pylori (24).

Sun et al (25) assessed the expression levels of E-cadherin by immunohistochemical analysis in 58 GC tissues, 40 adjacent tissues and 42 CNAG tissues. Sun et al (25) demonstrated that the expression levels of E-cadherin in specimens of patients with GC were significantly lower than those of patients with CNAG, which is consistent with the present findings. Furthermore, the present study highlighted that the changes in the expression levels of the EMT markers E-cadherin and $\mathrm{N}$-cadherin were initially noted at the IM stage of gastric adenocarcinoma formation, suggesting that EMT may contribute to the initiation of gastric adenocarcinoma. Subsequently, the specimens were divided into two groups according to $H$. pylori infection status and the data demonstrated that at the CNAG and GC stages, the expression levels of E-cadherin in the $H$. pylori-positive group were significantly lower than those in the H.pylori-negative group, while at the GC stage, the expression levels of $\mathrm{N}$-cadherin were significantly higher in the $H$. pylori-positive group than in the $H$. pylori-negative group. A recent study by Li et al (6) indicated that $H$. pylori infection reduces E-cadherin expression via the YY1 associated protein 1 signaling pathway in patients with CNAG, which is consistent with the results of the present study. These results strongly suggest that $H$. pylori infection initiates the EMT process at an early stage of gastric carcinogenesis, which may subsequently promote growth, metastasis and invasion of GC.

Subsequently, GES-1 cells were infected with $H$.pylori in vitro, which induced EMT, as detected by decreased E-cadherin expression and increased $\mathrm{N}$-cadherin expression in GES-1 cells in the early stage of $H$. pylori infection. In addition, the effect of $H$. pylori infection on EMT was further demonstrated by experiments in $H$. pylori-infected Mongolian gerbils. H. pylori infection induced a decrease in E-cadherin levels and an increase in $\mathrm{N}$-cadherin expression, which was consistent with the in vitro results.

Activation of the AKT signaling pathway is involved in the EMT process by directly affecting the expression of specific transcription factors, such as Twist, Snail and Slug, the expression of integrin-linked kinase and extracellular matrix or by interacting with other signaling pathways, such as the TGF- $\beta, N F-\kappa B$, Ras and Wnt/ $\beta$-catenin signaling pathways (13). Activation of AKT/GSK3 $\beta$ can increase the transcriptional activity and intracellular levels of $\beta$-catenin, which exerts a positive regulatory effect on the Wnt signaling pathway $(26,27)$. The present results indicated that the levels of p-AKT and p-GSK $3 \beta$ in GES-1 cells began to increase following 0.5 and $3 \mathrm{~h}$, respectively, of co-culture with $H$. pylori and that this trend of p-AKT persisted until the $9 \mathrm{~h}$ time point and the trend for $\mathrm{p}-\mathrm{GSK} 3 \beta$ persisted until the $12 \mathrm{~h}$ time point. Furthermore, GES-1 cells were pre-treated with the AKT inhibitor VIII or DMSO, which was used as a negative control. Following $1 \mathrm{~h}$ of co-culture with $H$. pylori, the protein expression levels of p-AKT, p-GSK3 $\beta, \mathrm{N}$-cadherin and E-cadherin in GES-1 cells that were pre-treated with the AKT inhibitor VIII did not change significantly compared with those noted in the cells that were not infected with H. pylori. These results indicated that inhibition of AKT activity blocked the development of EMT, demonstrating that the $H$. pylori-associated EMT process was AKT-dependent.

To further validate the role of the AKT/GSK $3 \beta$ signaling pathway in $H$. pylori-induced EMT, the levels of AKT, p-AKT, GSK $3 \beta$ and $\mathrm{p}-\mathrm{GSK} 3 \beta$ were examined in Mongolian gerbils 
with or without $H$. pylori infection. Although no significant differences were noted in the expression levels of AKT and GSK3 $\beta$ between the two groups, $H$. pylori infection activated phosphorylated AKT and phosphorylated GSK3 $\beta$. These results were consistent with the in vitro findings.

There were a few limitations of the present study. Although the role of the AKT/GSK3 $\beta$ signaling pathway in $H$. pylori-induced EMT was thoroughly examined, other signaling pathways involved in the mechanism underlying this process require further exploration. Although the present study focused on the role of the AKT/GSK $3 \beta$ signaling pathway, whether this signaling pathway interacts with other signaling pathways in $H$. pylori-induced EMT remains to be explored. In addition, there is still a gap between activation of AKT and EMT, and the exact mechanism by which the AKT/GSK3 $\beta$ signaling pathway regulates EMT is not clear. Future studies should investigate this gap in further.

In conclusion, the present study indicated that $H$. pylori infection activated AKT and resulted in the phosphorylation and inactivation of GSK $3 \beta$, which promoted early-stage EMT development. These effects were AKT-dependent and were blocked by application of the AKT inhibitor VIII.

\section{Acknowledgements}

The authors would like to thank Professor Jianzhong Zhang (Chinese Center for Disease Control and Prevention, Beijing, China) for providing the wild-type $H$. pylori $\mathrm{CagA}^{+} \mathrm{VaCA}^{+}$strain (ATCC43504; originally from American Type Culture Collection).

\section{Funding}

The present study was supported by grants from the National Natural Science Foundation of China (grant nos. 81060038, 81460377, 81670507, 81760106, 81870395, 81960112 and $81900500)$, the Natural Science Foundation of Jiangxi Province of China (grant nos. 20142BAB215036 and 20171BAB205012), the Jiangxi Provincial Department of Science \& Technology (grant no. 20192BAB215006) and the Subject of Jiangxi Provincial Department of Health (grant no. 202110024).

\section{Availability of data and materials}

The datasets used and/or analyzed during the current study are available from the corresponding author on reasonable request.

\section{Authors' contributions}

YO, GL, JH and NLu conceived and designed the study. YO and GL performed the experiments, and NLu analyzed the data. WX and ZY collected human specimens and analyzed immunohistochemical data. CX, CZ and JC provided assistance with analyses of the data and technical issues. YO, NLi and YZ interpreted the data and drafted the manuscript. NLu supervised the study. All authors read and approved the final manuscript.

\section{Ethics approval and consent to participate}

The use of human tissues was approved by the Human Ethics Committee of the First Affiliated Hospital of Nanchang
University. All patients signed an informed consent form for their participation in the study protocol. The First Affiliated Hospital of Nanchang University Ethics Committee approved all animal experiment protocols.

\section{Patient consent for publication}

Not applicable.

\section{Competing interests}

The authors declare that they have no competing interests.

\section{References}

1. Karimi P, Islami F, Anandasabapathy S, Freedman ND and Kamangar F: Gastric cancer: Descriptive epidemiology, risk factors, screening, and prevention. Cancer Epidemiol Biomarkers Prev 23: 700-713, 2014.

2. Malfertheiner P,Megraud F, O'Morain CA, Gisbert JP, Kuipers EJ Axon AT, Bazzoli F, Gasbarrini A, Atherton J, Graham DY, et al: Management of Helicobacter pylori infection-the maastricht V/florence consensus report. Gut 66: 6-30, 2017.

3. Graham DY: Helicobacter pylori update: Gastric cancer, reliable therapy, and possible benefits. Gastroenterology 148: 719-731.e3, 2015.

4. Watanabe T, Takahashi A, Suzuki K, Kurusu-Kanno M, YamaguchiK,Fujiki Hand Suganuma M:Epithelial-mesenchymal transition in human gastric cancer cell lines induced by TNF- $\alpha$-inducing protein of Helicobacter pylori. Int J Cancer 134: 2373-2382, 2014

5. Choi YJ, Kim N, Chang H, Lee HS, Park SM, Park JH, Shin CM, Kim JM, Kim JS, Lee DH and Jung HC: Helicobacter pylori-induced epithelial-mesenchymal transition, a potential role of gastric cancer initiation and an emergence of stem cells. Carcinogenesis 36: 553-563, 2015.

6. Li N, Feng Y, Hu Y, He C, Xie C, Ouyang Y, Artim SC, Huang D, Zhu Y,LuoZ, et al: Helicobacter pylori CagA promotes epithelial mesenchymal transition in gastric carcinogenesis via triggering oncogenic YAP pathway. J Exp Clin Cancer Res 37: 280, 2018.

7. Nieto MA, Huang RY, Jackson RA and Thiery JP: EMT: 2016. Cell 166: 21-45, 2016.

8. Kalluri R and Weinberg RA: The basics of epithelialmesenchymal transition. J Clin Invest 119: 1420-1428, 2009.

9. Wong SHM, Fang CM, Chuah LH, Leong CO and Ngai SC: E-cadherin: Its dysregulation in carcinogenesis and clinical implications. Crit Rev Oncol Hematol 121: 11-22, 2018.

10. Ye X and Weinberg RA: Epithelial-mesenchymal plasticity: A central regulator of cancer progression. Trends Cell Biol 25: 675-686, 2015.

11. Dongre A and Weinberg RA: New insights into the mechanisms of epithelial-mesenchymal transition and implications for cancer. Nat Rev Mol Cell Biol 20: 69-84, 2019.

12. Yang $J$ and Weinberg RA: Epithelial-mesenchymal transition: at the crossroads of development and tumor metastasis. Dev Cell 14: 818-829, 2008.

13. Xu W, Yang Z and Lu N: A new role for the PI3K/Akt signaling pathway in the epithelial-mesenchymal transition. Cell Adh Migr 9: 317-324, 2015.

14. Warfel NA and Kraft AS: PIM kinase (and Akt) biology and signaling in tumors. Pharmacol Ther 151: 41-49, 2015.

15. Beurel E, Grieco SF and Jope RS: Glycogen synthase kinase-3 (GSK3): Regulation, actions, and diseases. Pharmacol Ther 148: 114-131, 2015.

16. Katoh $\mathrm{M}$ and Katoh M: Cross-talk of WNT and FGF signaling pathways at GSK3beta to regulate beta-catenin and SNAIL signaling cascades. Cancer Biol Ther 5: 1059-1064, 2006.

17. Shu X, Yang Z, Li ZH, Chen L, Zhou XD, Xie Y and Lu NH: Helicobacter pylori Infection activates the akt-Mdm2-p53 signaling pathway in gastric epithelial cells. Dig Dis Sci 60: 876-886, 2015.

18. Yang Z, Xie C, Xu W, Liu G, Cao X, Li W, Chen J, Zhu Y, Luo S Luo $\mathrm{Z}$ and $\mathrm{Lu} \mathrm{N}$ : Phosphorylation and inactivation of PTEN at residues Ser380/Thr382/383 induced by Helicobacter pylori promotes gastric epithelial cell survival through PI3K/Akt pathway. Oncotarget 6: 31916-31926, 2015. 
19. Hamilton SR and Aaltonen LA: Pathology and genetics of tumours of the digestive system. IARC Press, Lyon, 2000.

20. Dixon MF, Genta RM, Yardley JH and Correa P: Classification and grading of gastritis. The updated sydney system. International workshop on the histopathology of gastritis, houston 1994. Am J Surg Pathol 20: 1161-1181, 1996.

21. Yang Z, Yuan XG, Chen J, Luo SW, Luo ZJ and Lu NH: Reduced expression of PTEN and increased PTEN phosphorylation at residue Ser380 in gastric cancer tissues: A novel mechanism of PTEN inactivation. Clin Res Hepatol Gastroenterol 37: 72-79, 2013

22. Xu W, Huang Y, Yang Z, Hu Y, Shu X, Xie C, He C, Zhu Y and Lu N: Helicobacter pylori promotes gastric epithelial cell survival through the PLK1/PI3K/Akt pathway. Onco Targets Ther 11: 5703-5713, 2018.

23. Suerbaum S and Michetti P: Helicobacter pylori infection. N Engl J Med 347: 1175-1186, 2002.

24. Naumann M, Sokolova O, Tegtmeyer N and Backert S Helicobacter pylori: A paradigm pathogen for subverting host cell signal transmission. Trends Microbiol 25: 316-328, 2017.
25. Sun GY, Wu JX, Wu JS, Pan YT and Jin R: Caveolin-1, E-cadherin and $\beta$-catenin in gastric carcinoma, precancerous tissues and chronic non-atrophic gastritis. Chin J Cancer Res 24: 23-28, 2012.

26. Sheng S, Qiao M and Pardee AB: Metastasis and AKT activation. J Cell Physiol 218: 451-454, 2009.

27. Qiao M, Sheng S and Pardee AB: Metastasis and AKT activation. Cell Cycle 7: 2991-2996, 2008

(i) (9) This work is licensed under a Creative Commons Attribution-NonCommercial-NoDerivatives 4.0 International (CC BY-NC-ND 4.0) License. 